\title{
Differential Response of Meloidogyne, Pratylenchus, Globodera, and Xiphinema Species to the Nematicide Fluazaindolizine
}

\author{
Catherine L. Wram ${ }^{1, \dagger}$ and Inga Zasada ${ }^{2}$ \\ ${ }^{1}$ Department of Botany and Plant Pathology, Oregon State University, Corvallis, OR 97331 \\ 2 U.S. Department of Agriculture-Agricultural Research Service Horticultural Crops Research Unit, Corvallis, OR 97330 \\ Accepted for publication 20 July 2020.
}

\begin{abstract}
This research focused on the effects of fluazaindolizine on a diversity of plant-parasitic nematodes. In microwell assays, 24-h dose-response curves were generated for several species and populations of Meloidogyne, Pratylenchus neglectus, P. penetrans, Globodera ellingtonae, and Xiphinema americanum. In a greenhouse study, the impact of fluazaindolizine on fecundity of $M$. incognita, M. hapla, and M. chitwoodi was tested by exposing nematodes for $24 \mathrm{~h}$ in solution and inoculating on tomato. The average 24-h $\mathrm{ED}_{50} \mathrm{~s}$ (dose that resulted in the immobility of $50 \%$ of exposed nematodes) for M. hapla, M. chitwoodi, and M. incognita were 325.7, 223.4, and $100.7 \mathrm{ppm}$, respectively. $M$. hapla had the most variation among populations, with 24-h $\mathrm{ED}_{50} \mathrm{~s}$ ranging from 72 to $788 \mathrm{ppm}$. G. ellingtonae had the lowest $24-\mathrm{hD}_{50}$ at $30 \mathrm{ppm}$. Pratylenchus spp. were unaffected by
\end{abstract}

ABSTRACT

Plant-parasitic nematodes are significant agricultural pests due to their vast host ranges and destructive life styles, which have negative effects on yields and quality of annual and perennial crops. Cyst (Globodera spp.) and root-knot (Meloidogyne spp.) nematodes stand out as two of the most damaging plant-parasitic nematode genera due to their endoparasitic life-style and ability to manipulate host cells (Jones et al. 2013). Globodera spp. are considered quarantine pests throughout the world; in the U.K. alone, they are responsible for $9 \%$ loss of potato yield each year (Moxnes and Hausken 2007). G. ellingtonae, while demonstrated to have limited to no effect on potato yield (Zasada et al. 2019), has a close phylogenetic relationship to the quarantine pests G. pallida and G. rostochiensis (Skantar et al. 2011), making it a good proxy for understanding the effects of nematicides on this genus of nematodes. Meloidogyne spp. are of great significance due to their global distribution and wide host range (Bernard et al. 2017; Perry and Moens 2013). This group of nematodes can cause yield loss in most major agricultural crops, up to $20 \%$ loss in solanaceous vegetables and wine grapes (Koenning et al. 1999; Zasada et al. 2012) and up to $10 \%$ loss in corn (Koenning et al. 1999). The group of migratory ectoparasites, Pratylenchus spp. also impact longevity and lifetime yield of perennial crops (Quero-Garcia et al. 2017) and are destructive

†Corresponding author: C. Wram; wramc@oregonstate.edu

Funding: Financial support was provided by Corteva Agriscience and by the U.S. Department of Agriculture-Agricultural Research Service Current Research Information System (2072-22000-043-00D).

Mention of trade names or commercial products in this publication is solely for the purpose of providing specific information and does not imply recommendation or endorsement by the U.S. Department of Agriculture.

*The $\boldsymbol{e}$-Xtra logo stands for "electronic extra" and indicates that one supplementary table is published online.

The author(s) declare no conflict of interest.

(C) 2020 The American Phytopathological Society fluazaindolizine. $X$. americanum was the only species where effects of fluazaindolizine were reversible, but had a $24-\mathrm{ED}_{50}$ that fell in the range of the Meloidogyne spp. In the greenhouse study, M. chitwoodi was the least sensitive with reproduction reaching $62 \%$ of the untreated control after a pre-exposure to $47 \mathrm{ppm}$, whereas $M$. incognita and M. hapla at the same exposure dose had reproduction rates of 27 and $36 \%$ of the untreated control, respectively. Despite varying in in vitro responses to fluazaindolizine, reproduction of all Meloidogyne spp. was suppressed after only $24 \mathrm{~h}$ of exposure. This study expanded our understanding of how G. ellingtonae, $P$. thornei, $P$. penetrans, and $X$. americanum respond to fluazaindolizine.

Keywords: disease control and pest management, nematology in row crops, where they have been reported to reduce yield of wheat up to $85 \%$ (Nicol et al. 2003). Another group of migratory ectoparasites of global importance are Xiphinema spp. This group of nematodes cause root damage and inhibits nutrient and water uptake. Some species also vector plant viruses such as Tobacco ringspot virus and Tomato ringspot virus (Perry and Moens 2013; Wang et. al 2002). Due to the importance of these plant-parasitic nematodes, species and populations within these genera were the focus of this current study.

Management of plant-parasitic nematodes has changed over the past few decades because of constraints placed on chemical controls to reduce potential risks to the environment and human health (Duniway 2002; EPA 2008; Martin 2003; Zasada et al. 2010). This push for more environmentally and applicator friendly management strategies has led to the development of new chemical controls like fluazaindolizine, Salibro (Lahm et al. 2017). Fluazaindolizine, an imidazopyridine is not yet registered for widespread use. The modeof-action is currently unknown for fluazaindolizine, however, Lahm et al. (2017) found no evidence to support a mode-of-action similar to that of carbamates or organophosphates. Toxicity of this compound seems to be plant-parasitic nematode specific. When Caenorhabditis elegans adults and Drosophila melanogaster egg and adult stages were exposed to 200 and $300 \mathrm{ppm}$ of fluazaindolizine, immobility or mortality were not observed (Lahm et al. 2017). The bacteria feeding nematode, Acrobeloides buetschlii was exposed to concentrations of fluazaindolizine up to $250 \mathrm{ppm}$ and hatching and movement remained unaffected after 5and 7-days exposure (Thoden and Wiles 2019).

Other studies have shown the short-term sensitivity of $M$. incognita, $M$. javanica, and $M$. hapla to fluazaindolizine in laboratory assays (Lahm et al. 2017; Thoden and Wiles 2019; Thoden et al. 2019; Wram and Zasada 2019). Thoden et al. (2019) observed population response differences in movement in an aqueous environment and ability to move through sand among five populations each of $M$. incognita and $M$. javanica after exposure to fluazaindolizine. In the same study, $M$. javanica was less sensitive to the effects of fluazaindolizine than $M$. incognita. The observance of differential population response is not limited to fluazaindolizine, 
and differential response was also observed when Meloidogyne spp. were exposed to oxamyl (Thoden et al. 2019). Differential response of Heterodera glycines populations to penetrate soybean roots was also observed after exposure to aldicarb (Gourd et al. 1993). Application of fluazaindolizine in a field setting has yielded results varying from full suppression of $M$. incognita reproduction to no effect on various hosts in many geographical locations (Becker et al. 2019; Hajihassani et al. 2019; Silva et al. 2019; Watson and Desaeger 2019), indicating population, and therefore genetic background, could potentially play a role in fluazaindolizine efficacy.

To determine if population has an impact on sensitivity to fluazaindolizine, dose-response curves were created for four populations of $M$. chitwoodi, 10 populations of M. hapla, and two populations of $M$. incognita. Additionally, a population each of M. incognita and M. chitwoodi and two populations of M. hapla were pre-exposed for $24 \mathrm{~h}$ to fluazaindolizine and inoculated onto a susceptible tomato cultivar to assess impacts on fecundity. Additionally, to expand the knowledge of which plant-parasitic nematode species are sensitive to fluazaindolizine, dose-response curves were generated for one population each of $G$. ellingtonae, $X$. americanum, and $P$. neglectus, and two populations of $P$. penetrans.

\section{MATERIALS AND METHODS}

Nematode collection. The plant-parasitic nematode populations used in this study are summarized in Table 1. For the Meloidogyne spp., they were either obtained directly from the field or from established cultures. To establish populations in culture, field soil was placed in a 1:1 pasteurized loam/sand mix in $184 \mathrm{~cm}^{3}$ clay pots and planted with a tomato 'Rutgers' seedling. If extracted second-stage juveniles (J2) or eggs were received, then the solution containing nematodes was added to the roots of tomato 'Rutgers'. Nematodes were allowed to develop for 10 to 15 weeks. To obtain Meloidogyne spp. J2, egg masses were hand-picked from tomato roots and placed on a $1.5 \mathrm{~cm}$ diameter plastic hatching chamber with a $30 \mu \mathrm{m}$ nylon mesh in a 40-ml beaker containing water (Zasada et al. 2006; Nitao et al. 1999). Hatched Meloidogyne spp. $\mathrm{J} 2$ were collected after 3 days and stored at $4^{\circ} \mathrm{C}$ until used, no more than 2 days. Similar to Meloidogyne spp., G. ellingtonae $\mathrm{J} 2$ were also used in assays. Second-stage juveniles were obtained by placing cysts on $30 \mu$ m nylon mesh in a 40 -ml beaker containing a $10 \%$ potato root exudate solution (Kroese et al. 2011). Nematodes were collected after 7 days and stored at $4^{\circ} \mathrm{C}$ until used, no more than 2 days. For X. americanum and Pratylenchus spp., mixed stage nematodes were used. $X$. americanum was extracted from soil by decanting and sieving soil followed by sugar centrifugation with $1 \mathrm{M}$ sucrose solution (Ingham 1994). Pratylenchus species were extracted from roots under intermittent mist for 3 to 5 days (Ingham 1994). An M. incognita population originally collected from grape (Vitis vinifera) in Parlier, CA was used in all experiments as a control because dose-response curves have already been established for this population (Wram and Zasada 2019).

Microwell dose-response assays. Formulated Salibro was used in all assays, which is $41.5 \%$ of the active ingredient fluazaindolizine. However, all concentrations shown are that of active ingredient. Doseresponse curves were generated for each plant-parasitic nematode species/population using a microwell assay system (Zasada et al. 2006). Nematodes were added to wells of a 24-well cell culture plate (Falcon; Corning, Corning, NY) in $100 \mu \mathrm{l}$ of water; $900 \mu \mathrm{l}$ of nematicide solution was then added to achieve the desired fluazaindolizine concentration. Number of nematodes added to wells varied based on species: for Meloidogyne spp. and G. ellingtonae, 50 to $60 \mathrm{~J} 2$ were added to each well; for Pratylenchus spp., 50 to 60 mixed stage nematodes were added to each well; and for $X$. americanum, 20 to 25 mixed stage nematodes were added to each well. A single plant-parasitic nematode species/ population was tested per plate that contained a water control and the $M$. incognita control at $94 \mathrm{ppm}$. In all assays, plant-parasitic nematodes were exposed to fluazaindolizine at 47, 94, 141, and $189 \mathrm{ppm}$. These concentrations were determined to provide a dose-response curve

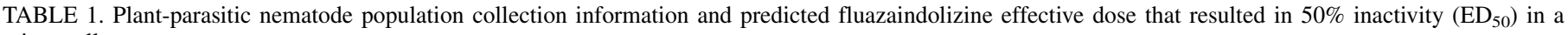
microwell assay system

\begin{tabular}{|c|c|c|c|c|c|c|c|c|}
\hline Species & Population $^{\mathrm{a}}$ & State & City & Original host & Stage tested & $\begin{array}{l}\text { 24-h ED } \mathrm{ED}_{50} \mathrm{ppm} \\
(95 \% \mathrm{CI})^{\mathrm{c}}\end{array}$ & $\begin{array}{l}\text { 24-h rinse } \mathrm{ED}_{50} \mathrm{ppm} \\
\quad(95 \% \mathrm{CI})^{\mathrm{d}}\end{array}$ & Citation $^{\mathrm{e}}$ \\
\hline \multirow{4}{*}{ Meloidogyne chitwoodi } & MC-CA-1 & $\mathrm{CA}$ & Tulelake & Potato & \multirow[t]{4}{*}{$\mathrm{J} 2^{\mathrm{b}}$} & $147(127-166)$ & $128(112-145)$ & Brown et al. 2009 \\
\hline & MC-WA-1 & WA & Prosser & Potato & & $136(121-152)$ & $113(98.1-128)$ & Brown et al. 2009 \\
\hline & MC-WA-2 & WA & Prosser & Potato & & $136(108-165)$ & $149(121-177)$ & Brown et al. 2009 \\
\hline & MC-WA-3 & WA & Quincy & Potato & & $474.1(196-752)$ & $167(144-191)$ & Mojtahedi et al. 1988 \\
\hline \multirow[t]{10}{*}{ M. hapla } & MH-CA-1 & $\mathrm{CA}$ & Davis & Tomato & \multirow[t]{10}{*}{$\mathrm{J} 2$} & $319(160-478)$ & $156(50-263)$ & \multirow[t]{10}{*}{ Liu and Williamson 2006} \\
\hline & MH-CT-1 & CT & Unknown & Ornamentals & & $132(93-172)$ & $116(98-134)$ & \\
\hline & MH-MI-1 & MI & Grant & Vegetables & & $491(50-931)$ & $174(126-221)$ & \\
\hline & MH-NY-1 & NY & Batavia & Tomato & & $283(199-367)$ & $102(46-158)$ & \\
\hline & MH-OR-1 & OR & Veneta & Grape & & $369(118-620)$ & $170(94-247)$ & \\
\hline & MH-WA-1 & WA & Prosser & $\begin{array}{l}\text { Concord } \\
\text { grape }\end{array}$ & & $789(35-1543)$ & $54(25-82)$ & \\
\hline & MH-WA-2 & WA & Mattawa & Grape & & $402(216-588)$ & $57(32-82)$ & \\
\hline & MH-WA-3 & WA & Alderdale & Grape & & $312(139-485)$ & $45(30-61)$ & \\
\hline & MH-WA-4 & WA & Paterson & Grape & & $88(68-108)$ & $35(21-48)$ & \\
\hline & MH-WA-5 & WA & Benton City & Grape & & $72(49-96)$ & $137(110-162)$ & \\
\hline \multirow[t]{2}{*}{ M. incognita } & MI-CA-1 & $\mathrm{CA}$ & Livingston & Grape & \multirow[t]{2}{*}{$\mathrm{J} 2$} & $150(79-220)$ & 44 (19-69) & \multirow{2}{*}{$\begin{array}{l}\text { Anwar et al. } 2002 \\
\text { Walker et al. } 1994\end{array}$} \\
\hline & MI-CA-2 & $\mathrm{CA}$ & $\begin{array}{l}\text { San Joaquin } \\
\text { Valley }\end{array}$ & Row crops & & $52(36-67)$ & $34(28-40)$ & \\
\hline Pratylenchus neglectus & PN-OR-1 & OR & Pendleton & Wheat & \multirow[t]{3}{*}{ Mixed stage } & N/A & N/A & \\
\hline P. penetrans & PP-WA-1 & WA & Lynden & Raspberry & & N/A & N/A & \\
\hline P. penetrans & PP-WA-2 & WA & Lynden & Raspberry & & N/A & N/A & \\
\hline Globodera ellingtonae & GE-OR-1 & OR & Powell Butte & Potato & $\mathrm{J} 2$ & $30(14-47)$ & $38(11-65)$ & Handoo et al. 2012 \\
\hline Xiphinema americanum & XA-WA-1 & WA & Woodburn & Grape & Mixed stage & $65(39-91)$ & $147(51-244)$ & \\
\hline
\end{tabular}


state.

b Second-stage juvenile (J2).

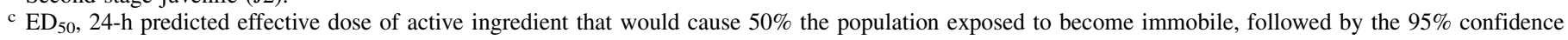
interval for the dose in parentheses.

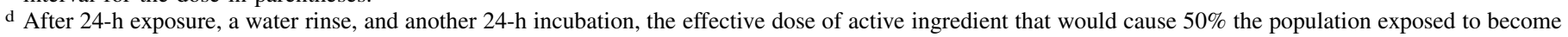
immobile (rinse $\mathrm{ED}_{50}$ ), followed by the $95 \%$ confidence interval for the dose in parentheses.

e Citation if a known population was used. 
resulting in 20 to $50 \%$ inactivity of $M$. incognita after $24 \mathrm{~h}$ of exposure in a previous study (Wram and Zasada 2019). Treatments were replicated four times per plate. The plate was then sealed with parafilm (Parafilm; Bemis Company, Neenah, WI), covered with foil, and incubated at room temperature for $24 \mathrm{~h}$. After $24 \mathrm{~h}$, nematodes were examined using an inverted microscope (Leica DM IL; Leica Microsystems, Wetzlar, Germany) at $100 \times$ magnification to enumerate the number of inactive and active nematodes. For all species besides $X$. americanum, active nematodes exhibited movement regardless if it was abnormal (twitching, sluggish, etc.), while straight nematodes that did not move were considered inactive. For $X$. americanum, inactive and active nematodes were determined by probing each nematode with a needle, with active nematodes being ones that responded to the probe and inactive nematodes having no movement when probed. To determine if effects of exposure were reversible, the fluazaindolizine solution was then removed using a pipette and replaced with $1 \mathrm{ml}$ of water. After another 24-h incubation period at room temperature, the number of active versus inactive nematodes were determined as described above. Each microwell assay was repeated at least two times to generate doseresponse curve for each plant-parasitic nematode species and population.

Statistical analyses were conducted using the software R (R Core Team 2017). The $\mathrm{R}$ package $d r c$ (Ritz et al. 2015) was used to generate dose-response curve models for each plant-parasitic nematode species and population. All data were Box-Cox transformed to correct for variance heterogeneity and nonnormality. A dose-response curve was fit to the transformed data using a threeparameter log-logistic model. Each model was then utilized to determine the effective dose needed to affect $50 \%$ of the treated nematodes $24 \mathrm{~h}$ after exposure $\left(24-\mathrm{h} \mathrm{ED}_{50}\right)$ and $24 \mathrm{~h}$ after removal of the compound (24-h rinse $\mathrm{ED}_{50}$ ) in the $d r c$ package.

Pre-exposure greenhouse assay. To understand if differential responses to fluazaindolizine observed in the microwell assays translated to differential effects on nematode fecundity, two populations of $M$. hapla, MH-WA-3 and MH-OR-1, M. chitwoodi MC-WA-3, and $M$. incognita MI-CA-1 were treated with fluazaindolizine and inoculated onto roots of tomato 'Rutgers'. Eggs were hatched as described above to obtain 1,500 Meloidogyne spp. J2 for each experimental replicate. Nematodes were suspended in $100 \mu \mathrm{l}$ of water and treated with $900 \mu \mathrm{l}$ of a fluazaindolizine solution in a 1.7- $\mathrm{ml}$ microcentrifuge tube for final exposure concentrations of 47, 94, 141, or 189 ppm of fluazaindolizine; a water control was included in all experiments. The microcentrifuge tubes were incubated at room temperature for $24 \mathrm{~h}$. After $24 \mathrm{~h}$, nematodes were rinsed with water on a $30 \mu \mathrm{m}$ mesh sieve for 5 to $10 \mathrm{~s}$ before being rinsed onto the roots of 2- to 3 -week-old tomato 'Rutgers' seedlings in a $10 \mathrm{~cm}$ pot containing a sand/loam mix (1:1) (Anderson Pots \#1007; Anderson Die \& Manufacturing Co., Portland, OR). Each fluazaindolizine concentration was repeated four times for each nematode species/population combination and the experiment was repeated. Inoculated plants were arranged in a randomized design in a greenhouse and grown under long-day conditions (16 h photoperiod) with $26 / 18^{\circ} \mathrm{C}$ day/night temperatures. After one week, plants were fertilized with a 9N-45P-15K plant starter fertilizer at the labeled rate (Jack's Professional Plant Starter; JR Peters Inc., Allentown, PA). Thereafter, plants were watered twice a week with $20 \mathrm{~N}-20 \mathrm{P}-20 \mathrm{~K}$ fertilizer at the labeled rate (JR Peters Inc.). After 10 weeks, plants were destructively harvested. The tops were removed and discarded, and roots were gently washed with water to remove adhering soil. Eggs were extracted by placing rinsed roots in $0.6 \%$ sodium hypochlorite and agitating at $300 \mathrm{rpm}$ for $3 \mathrm{~min}$. Roots were then rinsed on nested 250 and $25 \mu \mathrm{m}$ sieves, with eggs collected from the latter. Eggs were enumerated using an inverted microscope as described above.

Data were combined and analyzed by species. To examine the effects of fluazaindolizine on fecundity, the reproduction factor (RF) was calculated by dividing the final egg density $(P f)$ by the initial density of Meloidogyne spp. J2 (Pi) (Windham and Williams 1987). To normalize for variable reproduction rates of Meloidogyne spp., each treatment replicate RF value was divided by the average mean RF of the water control replicates for each species and population for that experimental replicate ([treatment replicate RF/ mean control RF for experiment/species/population] $\times 100=$ percentage $\mathrm{RF}$ of mean control RF). This ratio (RF ratio) indicated how well the nematodes were able to reach their maximum reproduction potential (control levels) in the experiment. This ratio was arcsine transformed to account for nonhomogeneous variability and nonnormal distribution of the data. A linear model was generated with factored concentrations and experimental replicate as explanatory variables using the R software (R Core Team 2017). To compare the impact of concentration on RF ratio, the previously described linear models were used as input for the $\mathrm{R}$ package 'lsmeans' (Lenth 2016) to determine least square means for each concentration, which were then Tukey corrected for multiple comparisons. An additional population parameter was added to the linear model for $M$. hapla to determine if the two populations examined in this study were affected differently by a pre-exposure to fluazaindolizine.

\section{RESULTS}

Modeling microwell dose-response assay data. A loglogistic regression model with four parameters was used to model the proportion of active Meloidogyne spp. J2 or mixed stages of other nematode species after 24-h exposure to fluazaindolizine, and $24 \mathrm{~h}$ after fluazaindolizine was removed and replaced with water (Supplementary Table S1). The four parameters in the log-logistic model are as follows: $b$, the coefficient denoting steepness of the dose-response curve; $c$ and $d$, the lower and upper asymptotes of the response curve; and $e$, the $\mathrm{ED}_{50}$ (Ritz et al. 2015). In all doseresponse curves for all species and populations tested, the threeparameter model was used where the lower asymptote, $c$, was fixed to 0 .

Response of Meloidogyne species populations to fluazaindolizine. Of the 16 Meloidogyne spp. populations tested, there was clear evidence of the nematicidal activity of fluazaindolizine against this genus. Even $24 \mathrm{~h}$ after the nematicide was replaced with water, all Meloidogyne spp. populations at each concentration tested had a further reduction in proportion of active $\mathrm{J} 2$ compared with proportion of active $\mathrm{J} 2$ at the same concentration 24 h earlier (Fig. 1). Of the Meloidogyne spp. populations evaluated, the $M$. incognita populations were the most sensitive to fluazaindolizine at $24 \mathrm{~h}$. At the lowest dose (47 ppm), both populations of $M$. incognita had $50 \%$ active $\mathrm{J} 2$ compared with the $M$. chitwoodi and M. hapla populations, which generally had $76 \%$ or more active $\mathrm{J} 2$ at the same dose. After $24 \mathrm{~h}$ of exposure at $189 \mathrm{ppm}$, the highest dose tested, this trend still held true. Both $M$. incognita populations had $>72 \%$ less active $\mathrm{J} 2$ compared with the control, whereas most M. hapla and M. chitwoodi populations had roughly $>60$ and $>50 \%$ less active J2 compared with the control, respectively (Fig. 1). The average 24-h $\mathrm{ED}_{50} \mathrm{~s}$ for $M$. hapla, $M$. chitwoodi, and $M$. incognita were 326,223 , and $101 \mathrm{ppm}$, respectively.

Of the four $M$. chitwoodi populations evaluated, three (MC-CA1, MC-WA-1, and MC-WA-2) had 24-h $\mathrm{ED}_{50}$ s that had overlapping $95 \%$ confidence intervals; however, the remaining population, MCWA-3, had a 24-h ED $\mathrm{ED}_{50}$ that was three times higher than that of the other M. chitwoodi populations (Table 1). M. hapla, with 10 populations tested, had the most variation in 24-h $\mathrm{ED}_{50}$ and 24-h rinse $\mathrm{ED}_{50}$, with $24-\mathrm{h} \mathrm{ED}_{50}$ ranging from 72 ppm to greater than 789 ppm (Table 1). This over 10-fold variation was observed in populations that were collected from similar geographical locations (south eastern Washington State) and from geographically disparate locations. Of the M. hapla populations, MH-WA-4 and MH-WA-5 were the most sensitive, with the two lowest 24-h $\mathrm{ED}_{50} \mathrm{~s}$. The 24-h $\mathrm{ED}_{50}$ for M. hapla MH-WA-5 was less than half that of the 24-h $\mathrm{ED}_{50}$ determined for $M$. hapla populations from MI, NY, CT, OR, and $\mathrm{CA}$. 
In a previous study, a $M$. incognita population from Parlier, CA was predicted to have a 24-h $\mathrm{ED}_{50}$ of $74 \mathrm{ppm}$ for fluazaindolizine (Wram and Zasada 2019). This population was used as a control throughout this study. Of the two additional populations of $M$. incognita tested, MI-CA-1 had a $24-\mathrm{h} \mathrm{ED}_{50}$ that was $2 \times$ that of the control $M$. incognita population. The other population, MICA-2 had a 24-h $\mathrm{ED}_{50}$ only 22 ppm lower than the control population. Despite having the higher $24-\mathrm{ED}_{50}$, MI-CA-1 was more sensitive at higher doses of fluazaindolizine than MI-CA-2 (Fig. 1).

Two Pratylenchus spp. were evaluated in this study, one population of $P$. neglectus and two populations of $P$. penetrans. All three of these populations were unaffected by fluazaindolizine at the concentrations tested, with the log-logistic regression model unable to determine either a $24-\mathrm{h} \mathrm{ED}_{50}$ of a $24-\mathrm{h}$ rinse $\mathrm{ED}_{50}$ for any of the populations (Table 1). After $24 \mathrm{~h}$ of exposure to fluazaindolizine, between 0 to 3\% of Pratylenchus spp. were less active than the control (Fig. 1). After fluazaindolizine was removed and $P$. neglectus rinsed, there was a slight decrease in the percentage of active mixed stage nematodes compared with the control, 84 to $85 \%$ at all concentrations tested. The two $P$. penetrans populations maintained the 0 and $3 \%$ less active nematodes compared with the control $24 \mathrm{~h}$ after fluazaindolizine was replaced with water (Fig. 1).

One population of $G$. ellingtonae was examined in this study. This nematode had the lowest $24-\mathrm{h} \mathrm{ED}_{50}$ of all the genera tested, $30 \mathrm{ppm}$ (Table 1). This $24-\mathrm{h} \mathrm{ED}_{50}$ was $>8 \times$ lower than the average for all the Meloidogyne spp. (625 ppm). There was a clear trend of true nematicidal activity of fluazaindolizine on G. ellingtonae as even after fluazaindolizine was removed, G. ellingtonae $\mathrm{J} 2$ were still only 27 and $20 \%$ as active as the control at 141 and $189 \mathrm{ppm}$, respectively. However, this was higher than the activity rates compared with the control after just $24 \mathrm{~h}$ of exposure, 16 and $14 \%$ for 141 and 189 ppm fluazaindolizine, respectfully.

The $X$. americanum population evaluated was the only nematode genera to have some reversibility to the effects of fluazaindolizine exposure. After $24 \mathrm{~h}$ of exposure, only 18 and $5 \%$ of $X$. americanum were active compared with control at 141 and $189 \mathrm{ppm}$, respectively. However, once fluazaindolizine was removed and $X$. americanum was incubated in water for another $24 \mathrm{~h}$ activity did increase; at 141 and $189 \mathrm{ppm} X$. americanum were 57 and $40 \%$ as active as the control, respectively (Fig. 1).

Pre-exposure fecundity assay with Meloidogyne spp. A 24-h pretreatment of Meloidogyne spp. with fluazaindolizine was effective at reducing fecundity in all tested species. $M$. incognita, $M$. hapla, and $M$. chitwoodi all had the strongest reduction in fecundity at doses greater than 94 ppm (Fig. 2). At the lowest two doses tested (47 and $94 \mathrm{ppm}$ ), M. chitwoodi was the least sensitive of the three Meloidogyne species, with mean reproduction levels still reaching greater than $50 \%$ of the untreated control. Reproduction of $M$. chitwoodi still reached $25 \%$ of its reproduction potential (mean untreated control) at all doses tested. This was unlike $M$. incognita and M. hapla both of which never exceeded $15 \%$ of their reproduction potential at three of the four doses tested $(94,141$, and $189 \mathrm{ppm}$ ). M. hapla and M. incognita had levels of reproduction compared with the mean of their respective controls $>2 \times$ less than $M$. chitwoodi at 47 and $94 \mathrm{ppm}$. M. incognita was the most sensitive of the three species tested. At $47 \mathrm{ppm}, M$. incognita had a 3.3-fold reduction in reproduction compared with the control, whereas $M$. hapla and $M$. chitwoodi had a 2.1 and 1.5-fold reduction, respectively.

Two populations of $M$. hapla were examined in the greenhouse assay (MH-OR-1 and MH-WA-3) to determine if population impacted how nematode fecundity was affected by pre-exposure to fluazaindolizine. When an additional parameter for population was added to the linear model for RF ratio, reproduction of MH-OR-1 was not found to be statistically different from that of MH-WA-3 $(P=0.106)$.

\section{DISCUSSION}

Plant-parasitic nematodes are a major global agricultural pest responsible for $\$ 118$ billion in yield and quality loss each year (Bernard et al. 2017), making their management of paramount importance for global food security. Recent changes in tolerance for

\begin{tabular}{|c|c|c|c|c|c|c|c|c|c|}
\hline \multirow[b]{2}{*}{ Species } & \multirow[b]{2}{*}{ Population } & \multicolumn{4}{|c|}{$24 \mathrm{hrs}$} & \multicolumn{4}{|c|}{24 hrs After Water Rinse } \\
\hline & & $47 \mathrm{ppm}$ & $94 \mathrm{ppm}$ & $141 \mathrm{ppm}$ & $189 \mathrm{ppm}$ & $47 \mathrm{ppm}$ & $94 \mathrm{ppm}$ & $141 \mathrm{ppm}$ & $189 \mathrm{ppm}$ \\
\hline \multirow{4}{*}{ M. chitwoodi } & $M C-C A-1$ & & & & & & & & \\
\hline & $M C-W A-1$ & & & & & & & & \\
\hline & MC-WA-2 & & & & & & & & \\
\hline & $M C-O R-1$ & & & & & & & & \\
\hline \multirow{10}{*}{ M. hapla } & $M H-C A-1$ & & & & & & & & \\
\hline & $M H-C T-1$ & & & & & & & & \\
\hline & $M H-M I-1$ & & & & & & & & \\
\hline & MH-NY-1 & & & & & & & & \\
\hline & $M H-O R-1$ & & & & & & & & \\
\hline & $M H-W A-1$ & & & & & & & & \\
\hline & MH-WA-2 & & & & & & & & \\
\hline & MH-WA-3 & & & & & & & & \\
\hline & MH-WA-4 & & & & & & & & \\
\hline & MH-WA-5 & & & & & & & & \\
\hline \multirow{2}{*}{ M. incognita } & $M I-C A-1$ & & & & & & & & \\
\hline & MI-CA-2 & & & & & & & & \\
\hline P. neglectus & $P N-O R-1$ & & & & & & & & \\
\hline P. penetrans & $P P-W A-1$ & & & & & & & & \\
\hline & $P P-W A-2$ & & & & & & & & \\
\hline G. ellingtonae & GE-OR-1 & & & & & & & & \\
\hline$X$. americanum & $X A-W A-1$ & & & & & & & & \\
\hline
\end{tabular}

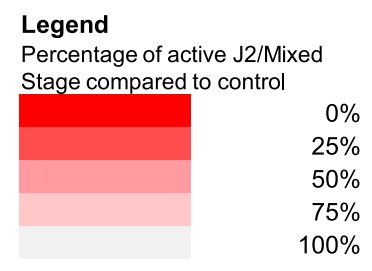

Fig. 1. Heatmap of mixed stage (Xiphinema americanum and Pratylenchus spp.) or second-stage juveniles (Meloidogyne spp. and Globodera ellingtonae) activity

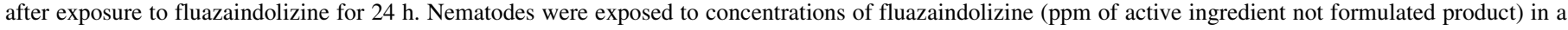

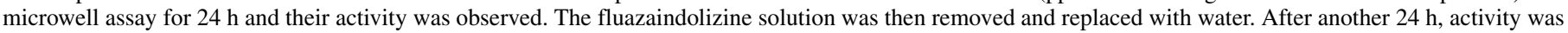

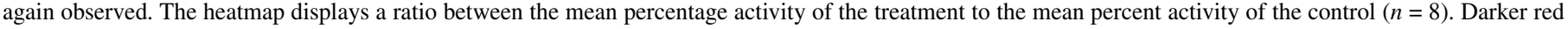
indicates the plant-parasitic nematode was less active after exposure to fluazaindolizine compared with a water control. 
environmental inputs and applicator safety have resulted in the ban or increased regulation of some chemicals, and the creation of new chemicals for the management of plant-parasitic nematodes (Faske and Hurd 2015; Kearn et al. 2014; Zasada et al. 2010). Fumigant and nonfumigant nematicides with warning or danger label designations have been heavily relied on due to their broad-spectrum efficacy against weeds, soilborne pathogens and plant-parasitic nematodes (Zasada et al. 2010). Newer nematicides have lower toxicity than traditional nematicides with caution label designations (Desaeger and Watson 2019). Due to the reduced risk nature of new nematicides, there is the potential for variable effects against a diversity of nematodes as compared with traditional nematicides that were broad spectrum in nature. Therefore, the aim of this study was to expand the knowledge of what plant-parasitic nematode genera and species may be susceptible to fluazaindolizine and to understand if species geographical location (population) impacts susceptibility to fluazaindolizine. We found that within the genus Meloidogyne both species and populations within species had a large range of tolerance, up to 10-fold, to a 24-h exposure of fluazaindolizine. Dose-response curves for fluazaindolizine were also determined for $G$. ellingtonae and X. americanum. Both of these species showed susceptibility to fluazaindolizine, while the two species of the Pratylenchus spp. evaluated did not.

Fluazaindolizine had clear irreversible effects on all Meloidogyne spp. and populations evaluated. This trend of irreversible effects on Meloidogyne spp. has been observed in other studies. Movement of $M$. incognita was reduced by 58 to $87 \%$ after $24 \mathrm{~h}$ of exposure to $250 \mathrm{ppm}$ of fluazaindolizine (Thoden and Wiles 2019). The two populations of $M$. incognita tested in this study were slightly below or within this range after exposure to fluazaindolizine at $94 \mathrm{ppm}$, with activity ranging from 45 to $60 \%$. In another study, Thoden et al. (2019) observed immobility of five populations of $M$. incognita and $M$. javanica after $\mathrm{J} 2$ were exposed to fluazaindolizine at $50 \mathrm{ppm}$ for $24 \mathrm{~h}$, with immobility ranging from 6 to $56 \%$ and 23 to $48 \%$, respectively. Although this range in variability was not observed in this study, after $24 \mathrm{~h}$ of exposure to fluazaindolizine there was up to $19 \%$ difference in activity between the two populations of $M$. incognita at the same dose, with the lowest variability only $1 \%$ difference at $188 \mathrm{ppm}$. A larger range in inconsistency in responses of Meloidogyne spp. populations may be
A
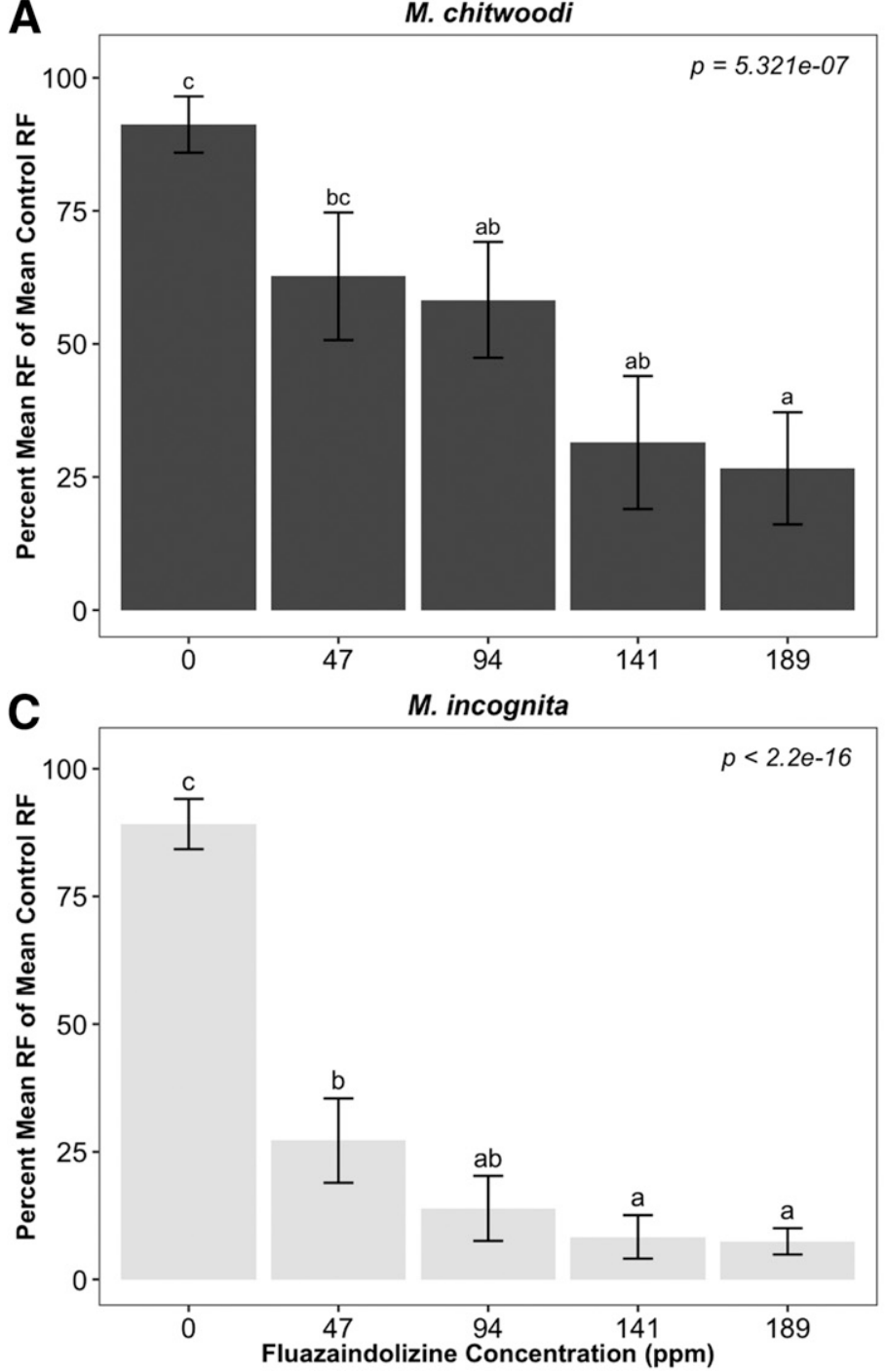

B M. hapla

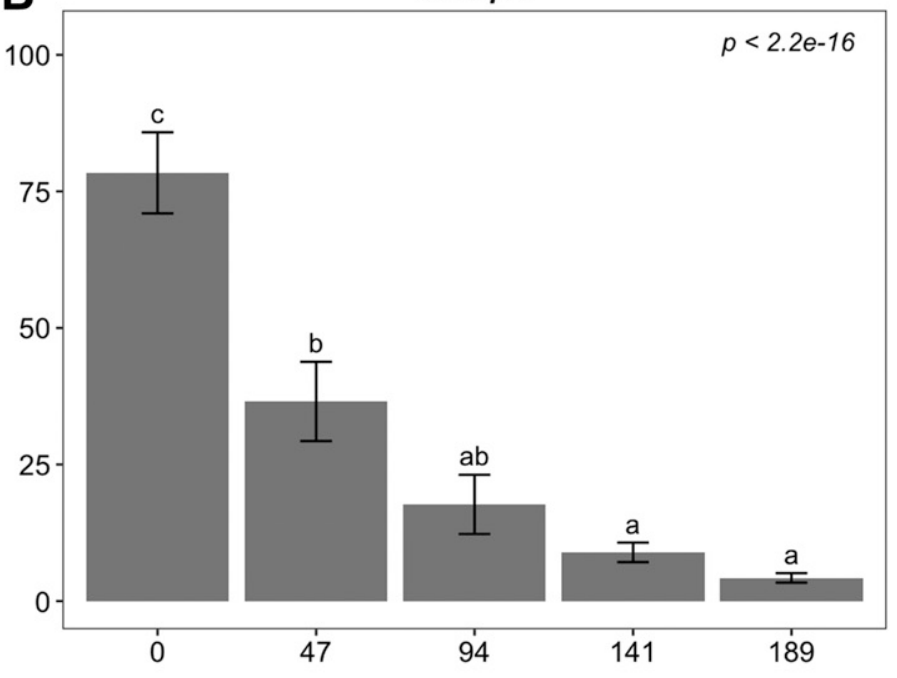

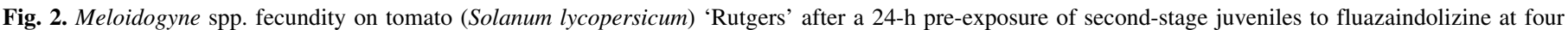

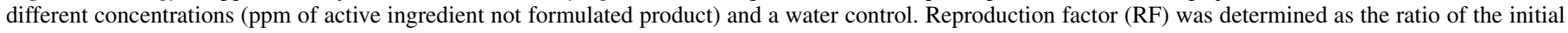

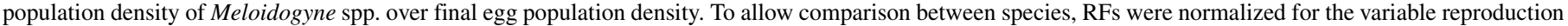

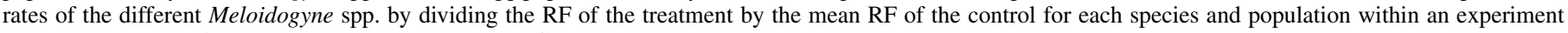

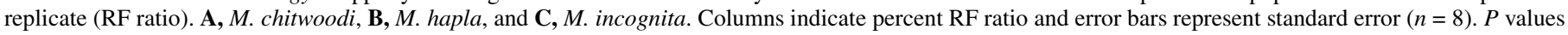

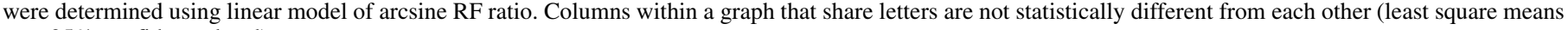
test, $95 \%$ confidence level). 
inherent to fluazaindolizine. This is in comparison with oxamyl with immobility of five $M$. incognita and $M$. javanica populations after $24 \mathrm{~h}$ of exposure to $25 \mathrm{ppm}$ ranging from 78 to $98 \%$ and 80 to $99 \%$, respectively, a much more consistent response across species than observed for fluazaindolizine (Thoden et al. 2019). In our study, the same inconsistency in response to fluazaindolizine was observed across the three species tested, $M$. incognita, M. chitwoodi, and $M$. hapla. At $47 \mathrm{ppm}$, the lowest dose tested, percentage of active $\mathrm{J} 2$ ranged from 48 to $67 \%, 78$ to $98 \%$, and 54 to $98 \%$ for $M$. incognita, $M$. chitwoodi, and $M$. hapla, respectively. This variability remained even after the compound was removed and replaced with water for another $24 \mathrm{~h}$ with percentage of active $\mathrm{J} 2$ ranging from 40 to $37 \%, 78$ to $97 \%$, and 40 to $91 \%$ at the $47 \mathrm{ppm}$ dose for $M$. incognita, $M$. chitwoodi, and M. hapla, respectively.

Thoden and Wiles (2019) evaluated the effects of a 24-h preexposure to 50 and $250 \mathrm{ppm}$ of fluazaindolizine on the ability of M. incognita and M. hapla, respectfully, to invade and cause galling on susceptible hosts. $M$. incognita pre-exposed to $50 \mathrm{ppm}$ of fluazaindolizine had reduced number of nematodes in roots 7 days after inoculation compared with the control (Thoden and Wiles 2019). Although in our study we did not measure nematode invasion, we found that a 24 -h pre-exposure to the lowest dose tested (47 ppm) was able to reduce reproduction to only approximately 25 to $30 \%$ of that of the control for both M. hapla and M. incognita. $M$. chitwoodi was less sensitive to fluazaindolizine, with these levels of suppression observed only at the two highest doses tested. Thoden and Wiles (2019) also reported similar sensitivity of M. hapla to fluazaindolizine. Meloidogyne hapla $\mathrm{J} 2$ pre-exposed to $250 \mathrm{ppm}$ of fluazaindolizine for $24 \mathrm{~h}$ had reduced ability of to invade roots, along with a reduction in root-galling scores from 5.8 to 3 at 21 days postinfection.

There have been a number of studies recently evaluating the efficacy of fluazaindolizine on various Meloidogyne spp. in different cropping systems in field, greenhouse, and microplot studies. There was a greater than twofold reduction in $M$. incognita reproduction compared with the control with both half and full rates of fluazaindolizine on tomato (Silva et al. 2019). In a microplot study with cucumber, even with starting populations of 20,000 $M$. incognita $\mathrm{J} 2$, fluazaindolizine was able to reduce the gall index by half (Hajihassani et al. 2019). In our previous study (Wram and Zasada 2019), fluazaindolizine at $94 \mathrm{ppm}$ applied as a drench eliminated $M$. incognita reproduction on susceptible tomato in a greenhouse study. These results are consistent with the greenhouse experiment performed here, in which $M$. incognita reproduction was consistently suppressed by exposure to fluazaindolizine even when exposure was just for $24 \mathrm{~h}$.

Watson and Desaeger (2019) found that fluazaindolizine at $1.12 \mathrm{~kg} / \mathrm{ha}$ did not affect population densities of M. hapla in several strawberry field trials. This is inconsistent with the results from our study. While not as sensitive as $M$. incognita, $M$. hapla reproduction rates were consistently reduced between 3 - and 12 -fold of that of the control. Additionally, there was no difference in suppression of reproduction of the two $M$. hapla populations evaluated by a pretreatment with fluazaindolizine. As these results are inconsistent with field evaluation of this compound, further field evaluation of M. hapla from multiple geographical locations and in several different cropping systems may provide more clarity as to the usefulness of fluazaindolizine in managing M. hapla. Further investigation into the impacts of fluazaindolizine on $M$. chitwoodi outside of greenhouse experiments is also necessary as this species was the least sensitive to the 24-h pre-exposure of fluazaindolizine.

$P$. penetrans and $P$. neglectus were unaffected by fluazaindolizine at any of the concentrations evaluated, up to $189 \mathrm{ppm}$. These results are consistent with a field experiment conducted in strawberry where fluazaindolizine drip applied at $1.12 \mathrm{~kg} / \mathrm{ha}$ at the time of strawberry transplant failed to suppress $P$. penetrans (Watson and Desaeger 2019). Further evaluation of other important Pratylenchus species and their sensitivity to fluazaindolizine are necessary to determine if this compound could be effective for the management of other species in this genus in other cropping systems.

There have been no reported evaluations of fluazaindolizine either in vitro or in the field for the management for either G. ellingtonae or X. americanum. Both of these species were very sensitive to fluazaindolizine in the microwell assays, making fluazaindolizine a new potential management strategy for cropping systems impacted by these nematode species. Further experiments exploring how fluazaindolizine might impact nematode reproduction would be an important stepping stone before deploying this compound for use in the field.

Although the populations of the three different Meloidogyne spp. examined in this study showed a large range in fluazaindolizine sensitivity in in vitro assays, reproduction on a susceptible host was clearly suppressed in all species. The level of suppression did vary between these species and with the mode-of-action unknown for this compound, it is unclear how this sensitivity is conferred in each species and whether this could lead to some populations of Meloidogyne spp. not responding to fluazaindolizine in the field. This study also determined 24-h $\mathrm{ED}_{50} \mathrm{~s}$ for G. ellingtonae or $X$. americanum, which were in general more sensitive to fluazaindolizine than any Meloidogyne spp. tested. These differing levels of tolerance between species could lead to the need of more tailored application rates after further exploration in greenhouse or field experiments. Knowledge of the variable response of plantparasitic nematodes to fluazaindolizine could result in economic savings to growers and unnecessary chemical applications.

\section{LITERATURE CITED}

Anwar, S. A., McKenry, M., and Ramming, D. 2002. A search for more durable grape rootstock resistance to root-knot nematode. Am. J. Enol. Vitic. 53:19-23.

Becker, J. O., Ploeg, A., and Nuñez, J. J. 2019. Multi-year field evaluation of fluorinated nematicides against Meloidogyne incognita in carrots. Plant Dis. 103:2392-2396.

Bernard, G. C., Egnin, M., and Bonsi, C. 2017. The impact of plant-parasitic nematodes on agriculture and methods of control. in: Nematology-Concepts, Diagnosis and Control, InTech.

Brown, C. R., Mojtahedi, H., Zhang, L.-H., and Riga, E. 2009. Independent resistant reactions expressed in root and tuber of potato breeding lines with introgressed resistance to Meloidogyne chitwoodi. Phytopathology 99:1085-1089.

Desaeger, J. A., and Watson, T. T. 2019. Evaluation of new chemical and biological nematicides for managing Meloidogyne javanica in tomato production and associated double-crops in Florida. Pest Manag. Sci. 75: 3363-3370.

Duniway, J. M. 2002. Status of chemical alternatives to methyl bromide for pre-plant fumigation of soil. Phytopathology 92:1337-1343.

Faske, T. R., and Hurd, K. 2015. Sensitivity of Meloidogyne incognita and Rotylenchulus reniformis to fluopyram. J. Nematol. 47:316-321.

EPA. 2008. Fenamiphos; Amendment to Use Deletion and Product Cancellation Order. Federal Register. https://www.federalregister.gov/documents/ 2008/06/11/E8-13003/fenamiphos-amendment-to-use-deletion-and-product-cancellation-order

Gourd, T. R., Schmitt, D. P., and Barker, K. R. 1993. Differential sensitivity of Meloidogyne spp. and Heterodera glycines to selected nematicides. J. Nematol. 25:746-751.

Hajihassani, A., Davis, R. F., and Timper, P. 2019. Evaluation of selected nonfumigant nematicides on increasing inoculation densities of Meloidogyne incognita on cucumber. Plant Dis. 103:3161-3165.

Handoo, Z. A., Carta, L. K., Skantar, A. M., and Chitwood, D. J. 2012. Description of Globodera ellingtonae n. sp. (Nematoda: Heteroderidae) from Oregon. J. Nematol. 44:40-57.

Ingham, R. 1994. Nematodes. Pages 459-490 in: Methods of Soil Analysis Part 2, Microbiological and Biochemical Properties. R. W. Weaver, S. Angle, P. Bottomley, D. Bezdicek, and S. Smith, eds. Soil Science Society of America, Madison, WI.

Jones, J. T., Haegeman, A., Danchin, E. G. J., Gaur, H. S., Helder, J., Jones, M. G. K., et al. 2013. Top 10 plant-parasitic nematodes in molecular plant pathology. Mol. Plant Pathol. 14:946-961.

Kearn, J., Ludlow, E., Dillon, J., O'Connor, V., and Holden-Dye, L. 2014. Fluensulfone is a nematicide with a mode of action distinct from anticholinesterases and macrocyclic lactones. Pestic. Biochem. Physiol. 109:44-57. 
Koenning, S. R., Overstreet, C., Noling, J. W., Donald, P. A., Becker, J. O., and Fortnum, B. A. 1999. Survey of crop losses in response to phytoparasitic nematodes in the United States for 1994. J. Nematol. 31:587-618.

Kroese, D., Zasada, I. A., and Ingham, R. E. 2011. Comparison of Meldola's Blue staining and hatching assay with potato root diffusate for assessment of Globodera sp. egg viability. J. Nematol. 43:182-186.

Lahm, G. P., Desaeger, J., Smith, B. K., Pahutski, T. F., Rivera, M. A., Meloro, T., et al. 2017. The discovery of fluazaindolizine: A new product for the control of plant parasitic nematodes. Bioorg. Med. Chem. Lett. 27: $1572-1575$.

Lenth, R. V. 2016. Least-squares means: The R package lsmeans. J. Stat. Softw. 69:1-33.

Liu, Q. L., and Williamson, V. M. 2006. Host-specific pathogenicity and genome differences between inbred strains of Meloidogyne hapla. J. Nematol. 38:158-164.

Martin, F. N. 2003. Development of alternative strategies for management of soilborne pathogens currently controlled with methyl bromide. Annu. Rev. Phytopathol. 41:325-350.

Mojtahedi, H., Santo, G. S., and Wilson, J. H. 1988. Host tests to differentiate Meloidogyne chitwoodi races 1 and 2 and M. hapla. J. Nematol. 20: 468-473.

Moxnes, J. F., and Hausken, K. 2007. The population dynamics of potato cyst nematodes. Ecol. Modell. 207:339-348.

Nicol, J., Rivoal, R., Taylor, S., and Zaharieva, M. 2003. Global importance of cyst (Heterodera spp.) and lesion nematodes (Pratylenchus spp.) on cereals: Distribution, yield loss, use of host resistance and integration of molecular tools. Nematol. Monogr. Perspect. 2:1-19.

Nitao, J. K., Meyer, S. L., and Chitwood, D. J. 1999. In-vitro assays of Meloidogyne incognita and Heterodera glycines for detection of nematodeantagonistic fungal compounds. J. Nematol. 31:172-183.

Perry, R. N., and Moens, M. 2013. Plant Nematology, 2nd Ed. CAB International, Oxfordshire, U.K.

Quero-Garcia, J., Iezzoni, A., Pulawska, J., and Lang, G. A. 2017. Cherries: Botany, Production and Uses. CABI, Wallingford, U.K.

R Core Team. 2017. R: A Language and Environment for Statistical Computing. https://www.R-project.org/

Ritz, C., Baty, F., Streibig, J. C., and Gerhard, D. 2015. Dose-response analysis using R. PLoS One 10:e146021.
Silva, J. de O., Loffredo, A., da Rocha, M. R., and Becker, J. O. 2019. Efficacy of new nematicides for managing Meloidogyne incognita in tomato crop. J. Phytopathol. 167:295-298.

Skantar, A. M., Handoo, Z. A., Zasada, I. A., Ingham, R. E., Carta, L. K., and Chitwood, D. J. 2011. Morphological and molecular characterization of Globodera populations from Oregon and Idaho. Phytopathology 101:480-491.

Thoden, T., Pardavella, I., and Tzortzakakis, E. 2019. In vitro sensitivity of different populations of Meloidogyne javanica and $M$. incognita to the nematicides Salibro ${ }^{\mathrm{TM}}$ and Vydate®. Nematology 21:889-893.

Thoden, T. C., and Wiles, J. A. 2019. Biological attributes of Salibro ${ }^{\mathrm{TM}}$, a novel sulfonamide nematicide. Part 1: Impact on the fitness of Meloidogyne incognita, M. hapla and Acrobeloides buetschlii. Nematology 21:625-639.

Walker, M. A., Ferris, H., and Eyre, M. 1994. Resistance in Vitis and Muscadina species to Meloidogyne incognita. Plant Dis. 78:1055-1058.

Wang, S., Gergerich, R. C., Wickizer, S. L., and Kim, K. S. 2002. Localization of transmissible and nontransmissible viruses in the vector nematode Xiphinema americanum. Phytopathology 92:646-653.

Watson, T. T., and Desaeger, J. A. 2019. Evaluation of non-fumigant chemical and biological nematicides for strawberry production in Florida. Crop Prot. 117:100-107.

Windham, G. L., and Williams, W. P. 1987. Host suitability of commercial corn hybrids to Meloidogyne arenaria and M. incognita. J. Nematol. 19: 13-16.

Wram, C. L., and Zasada, I. A. 2019. Short-term effects of sublethal doses of nematicides on Meloidogyne incognita. Phytopathology 109:1605-1613.

Zasada, I. A., Halbrendt, J. M., Kokalis-Burelle, N., LaMondia, J., McKenry, M. V., and Noling, J. W. 2010. Managing nematodes without methyl bromide. Annu. Rev. Phytopathol. 48:311-328.

Zasada, I. A., Ingham, R. E., Baker, H., and Phillips, W. S. 2019. Impact of Globodera ellingtonae on yield of potato (Solanum tuberosum). J. Nematol. 51:e2019-e2073.

Zasada, I. A., Klassen, W., Meyer, S. L., Codallo, M., and Abdul-Baki, A. A. 2006. Velvetbean (Mucuna pruriens) extracts: Impact on Meloidogyne incognita survival and on Lycopersicon esculentum and Lactuca sativa germination and growth. Pest Manag. Sci. 62:1122-1127.

Zasada, I. A., Riga, E., Pinkerton, J. N., Wilson, J. H., and Schreiner, R. P. 2012. Plant-parasitic nematodes associated with grapevines, Vitis vinifera, in Washington and Idaho. Am. J. Enol. Vitic. 63:522-528. 